\title{
Discriminações condicionais após treino de pareamento consistente de estímulos complexos
}

\author{
Grauben Assis \\ Marcelo Galvão Baptista \\ Olivia Misae Kato \\ Aline Beckmann de Menezes \\ Universidade Federal do Pará
}

\begin{abstract}
Resumo
O objetivo do presente estudo foi verificar a formação de discriminações condicionais através de um procedimento de treino por pareamento consistente de estímulos complexos. Na Fase 1, com quatro universitários, foi realizado o treino AF, BE e DC envolvendo estímulos modelos e de comparação simples e reforçamento diferencial explícito. Na Fase 2, houve o treino AB-E/F e AD-C/F, com modelos complexos e estímulos de comparação simples num formato de pareamento consistente. Após isso, os participantes foram expostos aos testes de transitividade e de equivalência. Na Fase 3, os treinos AB-E/F e AD-C/F foram desmembrados (simplificados) em AB-E; AB-F; AD-C; AD-F, e reaplicados os mesmos testes. Todos os participantes formaram as relações condicionais e dois deles formaram as relações emergentes, anterior e posterior ao desmembramento dos treinos. Os resultados sugerem que esse desmembramento foi um procedimento adequado para reverter o controle restrito de estímulo.
\end{abstract}

Palavras-chave: discriminação condicional; treino de pareamento consistente; estímulos complexos; universitários

\begin{abstract}
Conditional discriminations following consistent matching-to-sample training of complex stimuli. The aim of this study was to verify the formation of conditional discriminations through a consistent matching training procedure with complex sample stimuli. Four university students were trained in the formation of AF, BE e DC conditional relations using simple stimuli and explicit differential reinforcement (Phase 1). They were subjected to consistent matching-to-sample training on the $\mathrm{AB}-\mathrm{E} / \mathrm{F}$ and $\mathrm{AD}-\mathrm{C} / \mathrm{F}$ relations involving complex sample stimuli (Phase 2). These participants were given the transitive and equivalent tests. Training trials were then "dismembered" (simplified) from AB-E/F, and AD-C/F sequences to AB-E; AB-F; AD-C; AD-F sequences, and the students were again tested for transitivity and equivalence (Phase 3). All participants reached the learning criterion. Two of them demonstrated the emergent relations tested before and after simplified training, and demonstrated response stability. These results indicated that the dismembered procedure was appropriate for reversing the restricted stimulus control.
\end{abstract}

Keywords: conditional discrimination; consistency matching-to-sample training; complex stimuli; stimulus equivalence; college students

A literatura tem apontado a formação de classes de estímulos equivalentes após o treino de discriminações condicionais sem consequiências diferenciais em cada tentativa (ver estudos de Álvares, Assis, Sampaio, \& Esteves, 2001; Assis, Baptista, Damin, \& Álvares, 1997; Assis, Baptista, Kato, \& Alves, 2000; Baptista \& Assis, 1995; Barros, Baptista, \& Assis, 1998; Damin, Assis, \& Baptista, 1998). Nestes estudos, o procedimento característico utilizado foi o treino de pareamento consistente de estímulos simples: juntamente com o modelo eram apresentados um estímulo de comparação correto e consistente, e dois estímulos de comparação incorretos. Estes dois últimos mudavam a cada duas tentativas. O delineamento desses estudos constitui um procedimento alternativo aos tradicionais, nos quais são programadas conseqüências diferenciais imediatas e explícitas em cada tentativa de treino. Tal procedimento pode ser considerado alternativo, na medida em que a estrutura dos blocos de tentativas de treino tem sido programada para gerar uma linha de base comportamental estável e sensível, na ausência de conseqüências diferenciais imediatas, podendo ser empregado para a investigação paramétrica de algumas variáveis.

No estudo de Baptista e Assis (1995), a natureza dos estímulos mostrou-se uma variável importante que interferiu 
na formação de classes; isso foi indicado pelo desempenho dos participantes, e confirmado pelo seu relato verbal em entrevistas pós-sessões. Os demais estudos investigaram condições apropriadas e favoráveis que possibilitassem a formação de classes de estímulos equivalentes após o estabelecimento de discriminações condicionais também sem conseqüências diferenciais. Essas condições envolveram as seguintes manipulações experimentais, dentre outras: a antecipação de testes (ou seja, a aplicação de cada teste pré-requisito logo após o treino de cada relação condicional); a introdução do fading (apresentação dos estímulos modelo e de comparação consistente com um sombreamento cuja intensidade era diminuída gradativamente das tentativas iniciais às finais dos blocos de treino); o estabelecimento de uma história de responder dos participantes a estímulos usuais para verificar o seu efeito sobre o responder a estímulos não usuais; a expansão de classes de estímulos; o uso de estímulos contextuais.

Não obstante a relevância da natureza de estímulos, é importante verificar o efeito de outras variáveis na formação de classes de equivalência (cf. Stikeleather \& Sidman, 1990). Neste sentido, Álvares et al. (2001) verificaram se a apresentação de estímulos não usuais em fading, num formato de treino de pareamento consistente, facilitaria a formação de discriminações condicionais em crianças pré-escolares e se essas discriminações exibiriam também as propriedades da equivalência. Após a demonstração das relações condicionais no treino, dois dos quatro participantes do estudo formaram relações simétricas com estímulos usuais, porém nenhum formou equivalência. Esses resultados sugerem a necessidade de uma investigação mais rigorosa do treino de pareamento consistente, do fading ou dos efeitos interativos de ambos, associados ou não à antecipação dos testes.

$\mathrm{Na}$ tentativa de identificar outras condições sob as quais seria possível a formação de discriminações condicionais sem conseqüências diferenciais, além das programadas em estudos anteriores, Assis, Baptista, Kato e Cardoso (2003) realizaram um estudo com estudantes universitários, num formato de treino de pareamento consistente de estímulos simples (figuras usuais) com atraso no emparelhamento ao modelo. Os autores treinaram as relações condicionais $\mathrm{AB}$ e $\mathrm{AC}$, intercaladas aos testes de simetria $\mathrm{BA}$ e $\mathrm{CA}$, e de equivalência $\mathrm{BC}$ e CB. Dos participantes, sete demonstraram as relações condicionais treinadas, cinco demonstraram as relações de simetria e um formou as relações de equivalência.

Stromer, Mcllvane, Dube e Mackay (1993) já haviam demonstrado o estabelecimento de discriminações condicionais e a formação de classes de equivalência envolvendo estímulos complexos. Além da diferença quanto às consequiências diferenciais imediatas programadas para cada escolha neste estudo, as quais eram inexistentes no estudo de Assis et. al. (2003), houve diferença concernente ao tipo de estímulos experimentais (complexos, em Stromer et al., 1993 e simples, em Assis et al., 2003). Estímulos complexos, ao contrário de estímulos simples, são aqueles constituídos por mais de um elemento, cada um dos quais pode controlar a resposta de forma independente (Stromer, McIlvane, \& Serna, 1993) ${ }^{1}$.
A investigação e a demonstração da formação de classes de estímulos complexos em laboratório é importante e necessária (ver Dube, 1996). A formação destas classes sugere implicações práticas pela aproximação entre o ambiente artificial de laboratório e o ambiente natural. Em ambiente natural, é raro serem encontrados estímulos simples, porém estímulos complexos são abundantes (Stromer \& Stromer, 1990). Nem sempre é possível a formação de classes contendo estímulos complexos, devido ao controle restrito sobre a resposta, isto é, controle exercido por apenas um elemento do estímulo modelo, ao invés do controle por todos os elementos. O controle restrito de estímulo (também chamado seletividade perceptual ou atenção seletiva, conforme Lopes Junior, Costa, Gonsales, \& Golfeto, 2001, p. 402), pode ser evitado. Para isso, ao serem apresentados os elementos do estímulo modelo complexo a um participante, é necessário garantir que este emita resposta (de observação) a todos os elementos do modelo (Rehfeldt, Clayton, \& Hayes, 1998).

Dube e McIlvane (1999) tentaram impedir o estabelecimento do controle restrito de estímulo, ao identificar e estabelecer alguns pré-requisitos relacionados ao controle por todos os elementos do estímulo complexo. Para isso, estes autores utilizaram um procedimento que aumentasse o controle da resposta de observação por todos os componentes do modelo; denominaram-no de respostas diferenciais de observação (differential observing responses - DOR). Neste procedimento busca-se reduzir o controle restrito de estímulo, estabelecendo contingências que requeiram a discriminação de todos os componentes do modelo complexo. No procedimento DOR, o estímulo modelo complexo é apresentado inicialmente sozinho e, após a resposta ao mesmo, são apresentados os estímulos de comparação, sendo apenas um destes totalmente idêntico ao modelo $(\mathrm{S}+)$; por exemplo, o modelo $\mathrm{AB}$ e os estímulos de comparação $\mathrm{AX}, \mathrm{AB}$ e YB. Esse procedimento pode estabelecer a discriminação de todos os elementos do modelo.

Um estudo conduzido por Assis, Kato, Menezes e Lopes Junior (2000), com universitários de ambos os sexos, na faixa etária entre 18 e 22 anos, de diferentes cursos de graduação, buscou estender esta análise, procurando fortalecer o controle discriminativo exercido pelos elementos do estímulo modelo complexo com a introdução do procedimento DOR.

No estudo, houve três fases. O procedimento DOR foi introduzido na Fase II, após o treino da linha de base programado para a Fase 1. Nesse treino, as relações condicionais foram AF (A1F1; A2F2; A3F3), BE (B1E1; B2E2; B3E3) e DC (D1C1; D2C2; D3C3), com modelos e estímulos de comparação simples (constituídos por apenas um elemento ou componente), e utilização de consequiências diferenciais imediatas para acertos e erros (mensagens escritas na tela do microcomputador e sons reproduzidos eletronicamente). Os modelos e os estímulos de comparação eram formas usuais e não-usuais (apresentadas apenas com o contorno, isto é, sem preenchimento), e cores. No procedimento DOR, as relações condicionais treinadas foram $\mathrm{AB}-\mathrm{E} / \mathrm{F}$ (A1B1-E1/F1; A2B2-E2/F2; A3B3-E3/F3) e AD-C/F (A1D1-C1F1; A2D2$\mathrm{C} 2 \mathrm{~F} 2$; A3D3-C3/F3), nas quais os modelos eram complexos 
(com dois elementos), indicados pelas letras A e B; A e D, e os estímulos de comparação (cor e forma usual e não-usual) eram simples, indicados pelas letras E, F e C, F, dessas relações, respectivamente. Nesse procedimento, cada estímulo modelo (constituído por forma usual ou não-usual e por cor) permanecia o mesmo ao longo das tentativas, variando os estímulos de comparação (forma usual ou não-usual e cor - representada pelas letras $\mathrm{E}$ ou $\mathrm{F}$ e $\mathrm{C}$ ou F). O treino foi programado para que o controle sobre a escolha do estímulo de comparação considerado correto fosse exercido independentemente pelos elementos ou componentes do modelo (tendo em conta a relação estabelecida anteriormente). Por exemplo, no modelo AB, o elemento A controlava a escolha de $\mathrm{F}$, e o elemento $\mathrm{B}$ controlava a escolha de $\mathrm{E}$, não requerendo, necessariamente, a observação de ambos os elementos do modelo, mas sim a observação de um elemento de cada vez (resposta de observação diferencial). Esse controle ocorria em função da apresentação consistente de ambos os estímulos, ou seja, a apresentação de um elemento do modelo juntamente com o estímulo de comparação correto em todas as tentativas do bloco.

Após todos esses treinos, foram aplicados testes de transitividade, EF, FC, CF e FC, e de equivalência, BD e DB, seguidos da fase de remediação, Fase III. Nessa fase, foi programado o re-treino, sem a utilização de consequiências diferenciais imediatas, em três blocos de tentativas, de relações condicionais estabelecidas na Fase 1, nas quais o desempenho dos participantes indicasse que o critério não fora alcançado. Por exemplo, o re-treino das relações AB-E/ $\mathrm{F}$ e/ou AD-C/F.

Os resultados mostraram que quatro dos cinco participantes alcançaram o critério de acerto nos treinos das relações condicionais com modelos complexos, mesmo na ausência de conseqüências diferenciais. Os autores concluíram que a introdução do DOR possibilitou estabelecer relações condicionais e formar classes de estímulos equivalentes, embora tenha havido, em geral, uma pequena variabilidade no número de exposição aos blocos de tentativas (na formação da linha de base e na emergência das relações testadas).

Como continuidade ao estudo apresentado por Assis et al. (2000), o objetivo deste trabalho foi verificar a formação de relações condicionais por meio de um procedimento de treino de pareamento consistente de estímulos com modelos complexos e verificar se essas relações condicionais seriam também equivalentes.

\section{Método}

\section{Participantes}

Quatro universitários, sendo dois do sexo feminino (ASG e CCR) e dois do masculino (RMP e FCN) participaram do estudo. Os participantes foram recrutados por contato pessoal e receberam $\mathrm{R} \$ 10,00$ pela sua participação, no final do experimento, independentemente do seu desempenho na tarefa.

\section{Situação experimental e equipamento}

As sessões experimentais foram realizadas numa sala de pesquisa do Laboratório de Psicologia Experimental da Universidade Federal do Pará, monitoradas pelo experimentador, em uma sala adjacente, através de um vidro unidirecional. Foi utilizado um microcomputador - Pentium de $133 \mathrm{Mhz}$, com monitor VGART, 14 polegadas, policromático, com tela sensível ao toque - para a apresentação dos estímulos na tela, programação dos procedimentos de treino, de testes e registro das escolhas efetuadas pelos participantes. O software foi elaborado em linguagem Visual Basic 5.0.

\section{Estímulos}

Foram empregados em todas as fases do estudo, os seguintes conjuntos de estímulos: A e F - cores (vermelho, azul, amarelo, verde, roxo, marrom e cinza); B e C - figuras usuais; D e E - figuras não usuais (formas sem preenchimento). Os estímulos dos conjuntos A, B e D formavam modelos complexos; os estímulos dos demais conjuntos eram estímulos de comparação simples (ver Figura 1).
Estímulos

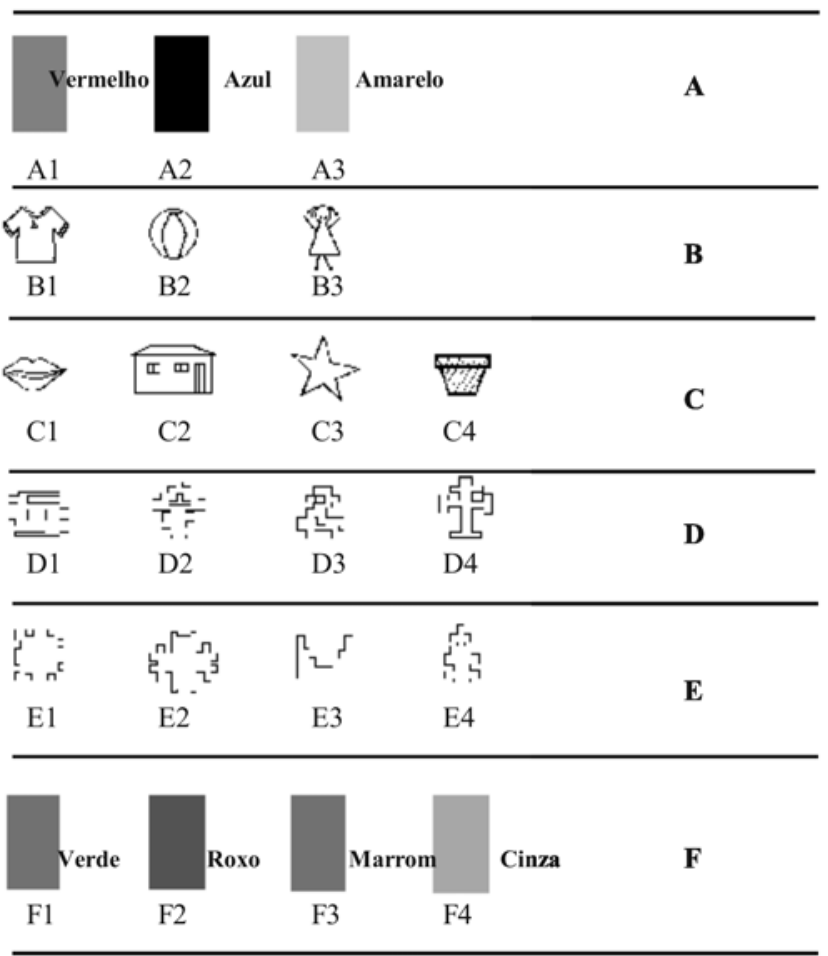

Nota:

Os estímulos dos conjuntos A, B e D são componentes dos modelos complexos. Os estímulos dos demais conjuntos são simples.

Os estímulos dos conjuntos A e F são usuais (cores).

Os estímulos dos conjuntos B e C são usuais (formas sem preenchimento). Os estímulos dos conjuntos D e E são não-usuais (formas sem preenchimento).

Figura 1. Estímulos utilizados no estudo. 


\section{Procedimento}

Foi utilizado o procedimento de emparelhamento arbitrário simultâneo com o modelo que envolvia três estímulos de comparação. A Figura 2, apresentada mais adiante, ilustra esse procedimento, com base no estudo de Dube e McIlvane (1999). Em cada tentativa o modelo era apresentado na "janela" central da tela; um toque no modelo não produzia o seu desaparecimento, mas gerava o aparecimento de três estímulos de comparação nas "janelas" laterais (uma outra "janela” permanecia desativada). Um toque adicional no modelo ou na "janela" desativada não produzia uma nova configuração dos estímulos na tela, porém, uma resposta a quaisquer estímulos de comparação gerava uma nova disposição dos estímulos na tela, em posições diferentes. O número de sessões por participante dependia do desempenho individual, sendo que a sessão durava no máximo sessenta (60) minutos. Foram programadas no máximo duas sessões por dia, intercaladas por um intervalo de pelo menos 15 minutos.

Foram programadas três fases para o estudo (ver Apêndice). Na Fase 1, com estímulos modelos simples, foram programadas consequiências diferenciais para acertos e erros. Cada resposta correta era seguida pela apresentação da mensagem, "Parabéns! Você acertou!", escrita no topo da tela, e de um sinal sonoro ("bip"). Após uma resposta incorreta, iniciava-se um intervalo entre tentativas de 1 segundo, durante o qual a tela embranquecia; ao término deste intervalo, uma nova configuração dos estímulos era apresentada. Não foram programados testes para esta fase. Ainda, na Fase I, as relações condicionais foram combinadas num único bloco. Na Fase II, com estímulos modelos complexos, não havia consequiências diferenciais para as respostas (ver detalhes adiante) e os blo$\cos$ de treino foram intercalados aos blocos de testes. A Fase III foi idêntica à Fase II, mas sem a combinação das relações condicionais num único bloco. Ou seja, houve, nessa fase, as tentativas de cada tipo programado (AB-E, AB-F, AD-C e AD-F), formavam blocos separados, portanto, como desmembramento dos treinos anteriores (AB-E/F e AD-C/F).

No início de cada sessão, havia uma folha de papel com uma instrução mínima em uma prancheta à frente do participante; a folha permanecia disponível para consulta durante a sessão. No final de cada sessão, aparecia automaticamente na tela a seguinte mensagem: "Por favor, chame o instrutor. Obrigado por participar do estudo". Foram fornecidas duas instruções mínimas: uma até o final da Fase 1 e outra a partir da Fase 2. As instruções foram as seguintes:

Instrução mínima 1 (no Treino - Fase 1): "Primeiro você tocará uma figura no centro da tela, então aparecerão outras em volta. Você deve tentar relacionar as figuras. Sempre que acertar, o percentual vai aumentar no contador; soará um "bip" e uma frase indicativa aparecerá no topo. Se errar, as figuras continuarão aparecendo. Você deve tentar acertar o máximo possível."

Instrução mínima 2 (no Treino e nos Testes - Fase 2) "Agora, você deve continuar tentando acertar a relação entre as figuras, mas você não saberá se acertou ou errou. O computador continuará registrando e você saberá os seus acertos no final do estudo."
O Apêndice apresenta um sumário do procedimento (os blocos de treino, a linha-de-base e os testes), o número e tipos de tentativas, bem como o tipo de conseqüência programada para as respostas.

\section{Pré-Treino}

No pré-treino, a distribuição dos estímulos de comparação nas "janelas” laterais era randomizada. Os estímulos eram apresentados apenas com o contorno preto, sobrepostos em janelas de fundo branco.

No pré-treino havia dois objetivos; o primeiro era familiarizar o participante com o equipamento e a situação experimental. Na primeira tentativa havia apenas uma figura como estímulo modelo (simples) e uma figura como estímulo de comparação. Na segunda e na terceira tentativas foram adicionados dois outros estímulos de comparação, um de cada vez, permanecendo o modelo simples. Na quarta tentativa foi acrescentado mais um componente ao estímulo modelo, passando este a ser complexo; isso constituía o segundo objetivo do pré-treino que foi preparar o participante para a situação posterior na qual o estímulo modelo seria apresentado com dois componentes.

\section{Fase I: treino com modelos simples}

Como mostrado no Apêndice, nesta fase, preliminar, foram programados 3 blocos de 12 tentativas (aquisição das discriminações condicionais) e 3 blocos de 2 tentativas (treinos mistos). Foram utilizados estímulos simples, com o objetivo de estabelecer um controle discriminativo para cada estímulo do modelo complexo que seria utilizado na fase seguinte, de modo a reduzir a probabilidade de ocorrência de controle restrito de estímulos.

As relações condicionais ensinadas foram: $\mathrm{AF}$ (A1F1; A2F2; A3F3), BE (B1E1; B2E2; B3E3) e DC (D1C1; D2C2; $\mathrm{D} 3 \mathrm{C} 3$ ). Após o treino de cada relação, o participante foi submetido ao treino misto (linha de base), envolvendo todas as relações já ensinadas. O critério de acerto foi de $100 \%$ e o número máximo de exposições ao bloco foi 10 .

\section{Fase II: treino com modelos complexos}

Nesta fase, as relações ensinadas foram as seguintes: AB-E/F (A1B1-E1/F1; A2B2-E2/F2; A3B3-E3/F3) e AD-C/F (A1D1-C1/F1; A2D2-C2/F2; A3D3-C3/F3).

O treino com modelos complexos foi uma adaptação do procedimento DOR (Dube \& McIlvane, 1999, conforme ilustrado na Figura 2). No procedimento DOR, é programada a apresentação randômica de dois conjuntos de estímulos de comparação. No presente estudo, os estímulos de comparação programados eram $\mathrm{E}$ ou $\mathrm{F}$ e $\mathrm{C}$ ou $\mathrm{F}$ (como indicam os tipos de tentativas da linha de base $\mathrm{AB}-\mathrm{E} / \mathrm{F}$ e AD-C/F, na Fase 2, $3^{\underline{a}}$ coluna do Apêndice). $\mathrm{O}$ estímulo modelo permanecia o mesmo ao longo das tentativas e poderia ocorrer o controle independentemente por um dos elementos do modelo, de acordo com a relação estabelecida na fase anterior (Fase 1). Os elementos do modelo eram "A" ou "B" ou "D", consoante a relação treinada anteriormente, sendo os estímulos de comparação, respectivamente, "E", "F" e "C". Neste procedimento, cada um dos elementos do estímulo modelo passa a controlar o responder separadamente. 
A

Pareamento ao modelo

com atraso

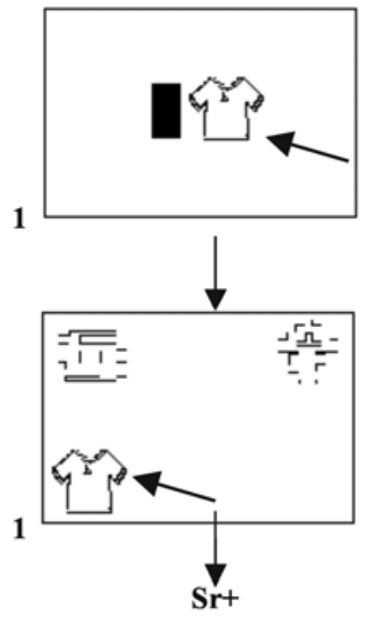

B

Pareamento ao modelo simultâneo

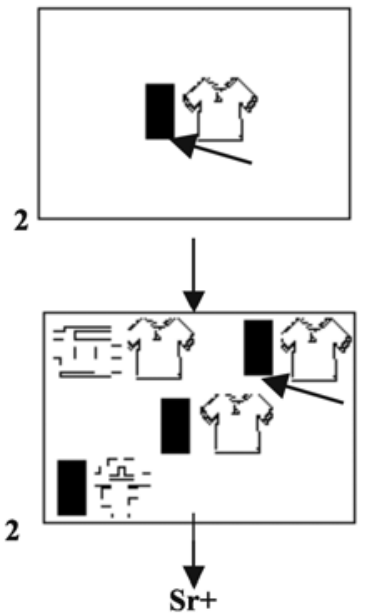

C

Procedimento DOR
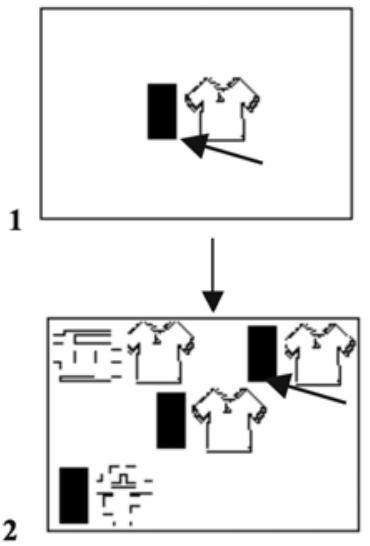

2

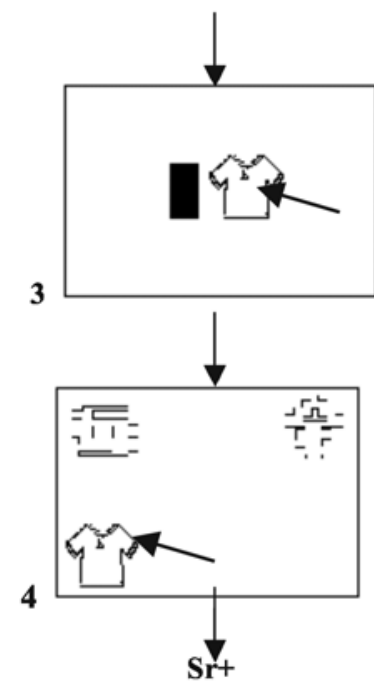

Nota: as "janelas" $\mathrm{C}(1,2,3$ e 4$)$ ilustram uma tentativa do procedimento DOR num formato de pareamento ao modelo com atraso. Em 1, o modelo (complexo) aparece sozinho na "janela" central; uma resposta (ver seta) ao elemento (esquerdo) do modelo produz o aparecimento, em 2, do mesmo modelo juntamente de estímulos de comparação (complexos) em "janelas" laterais; uma resposta ao elemento do estímulo de comparação idêntico ao modelo produz o reaparecimento deste sozinho, em 3; uma resposta ao outro elemento (direito) do modelo gera uma nova configuração, em 4, de estímulos de comparação simples, em posições diferentes, com ausência do modelo; uma resposta ao estímulo de comparação idêntico ao elemento do modelo complexo anterior produz uma consequiência reforçadora $(\mathrm{Sr}+)$. As "janelas" A e B ilustram, respectivamente, tentativas de treinos de pareamento ao modelo - com atraso e simultâneo -, envolvendo modelos complexos e consequiência reforçadora. Esses treinos antecederam o procedimento DOR.

Figura 2. Exemplo de uma tentativa do procedimento DOR (Dube \& Mcllvane, 1999) com adaptações.

Foi utilizado um treino de pareamento consistente para as relações $\mathrm{AB}-\mathrm{E} / \mathrm{F}$ e $\mathrm{AD}-\mathrm{C} / \mathrm{F}$. Neste treino eram apresentados, em cada tentativa, três estímulos de comparação, um dos quais previamente (Fase I) relacionado a um dos componentes do modelo complexo (Figura 1). Uma resposta a um dos componentes do modelo complexo produzia o aparecimento dos estímulos de comparação juntamente com o modelo. A resposta a quaisquer estímulos de comparação produzia, como única conseqüência não diferencial, a mudança na configuração dos estímulos na tela, isto é, o aparecimento do mesmo modelo complexo juntamente com os mesmos estímulos de comparação (formas ou cores) em posições diferentes, inclusive o de distração (assinalados no Apêndice com dois asteriscos). Por exemplo, a resposta ao componente cor (vermelha) do modelo complexo produzia o aparecimento de três cores: verde, roxa e marrom (ver coluna da direita da Figura 2). E a resposta ao componente forma (de camisa) desse mesmo modelo, produzia o aparecimento de três formas (codificadas como E1, E2 e E3) (ver coluna da esquerda da Figura 3). Após o ensino dessas relações condicionais (AB-E/F e AD-C/F), o participante era submetido aos respectivos treinos mistos com 12 tentativas. Caso o participante não atingisse o critério de 100\% de acertos, mesmo após o máximo de 10 exposições, ele seria submetido a um procedimento de correção. Este procedimento consistia nos respectivos treinos mistos A-F e B-E ou D-C por treino de pareamento consistente. Se atingisse o critério no procedimento de correção, o participante seria submetido novamente ao emparelhamento arbitrário com o modelo da respectiva relação, envolvendo o treino de pareamento consistente. Caso não alcançasse novamente o critério, seria então dispensado do estudo. 
1A)

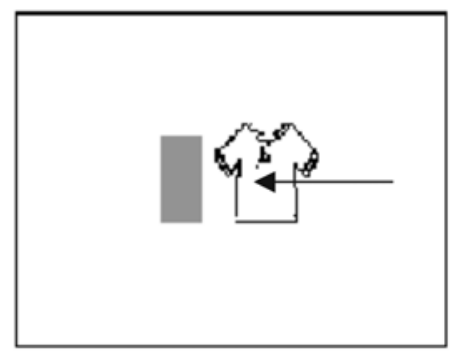

1b)

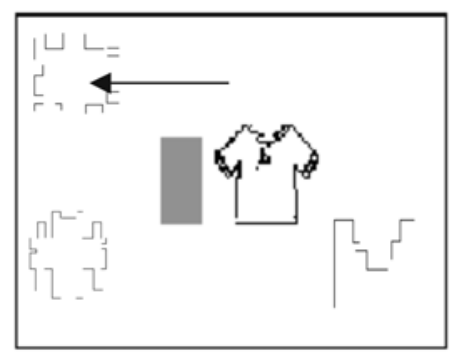

$2 \mathrm{a}$

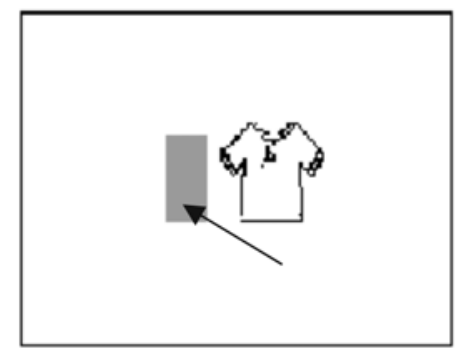

2B)

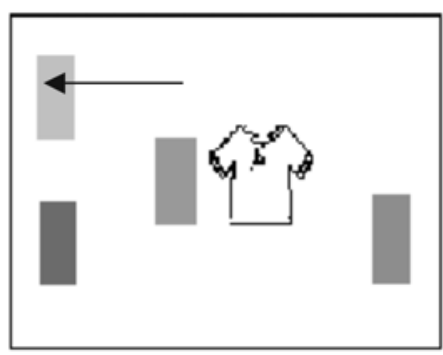

Nota: em 1A, a resposta (ver seta) a um componente (B1- figura de camisa) do modelo complexo (A1B1) produzia a apresentação (em 1B) dos estímulos de comparação (E1, E2 e E3 - figuras nãousuais, sem preenchimento), com a permanência do modelo. Em 2A, a resposta (ver seta) ao outro componente (A1- cor vermelha) do modelo produzia a apresentação dos estímulos de comparação (F1, F2 e F3 - cores verde, roxa e marrom, respectivamente), sem a remoção do modelo. Um desses estímulos (E1 ou F1, conforme indicação da seta) era consistente (Sc), ou seja, sempre pareado com o modelo, enquanto dois outros estímulos eram inconsistentes (Si), isto é, variando ao longo do treino. Esse tipo de treino com reexposição às tentativas aumentava a probabilidade do responder ao estímulo consistente, em detrimento da resposta aos estímulos inconsistentes.

Figura 3. Exemplo de uma tentativa do tipo A1B1 E1(+) E2E3 e do tipo A1B1 F1(+)F2F3 do Treino AB-E/F (ver Fase II no Apêndice).

\section{Testes de transitividade e equivalência}

Após a linha-de-base AB-E/F, foram aplicados os testes EF (transitividade) FE (equivalência), e após a linha-de-base $\mathrm{AD}-\mathrm{C} / \mathrm{F}$, os seguintes testes: $\mathrm{CF}$ (transitividade) e $\mathrm{FC}, \mathrm{BD}, \mathrm{e}$ DB (equivalência). Em todos os testes, exigia-se $100 \%$ de acertos e havia no máximo duas exposições. Terminada a última sessão experimental, foi realizada uma entrevista com cada participante, na qual ele (ou ela) era questionado(a) a respeito de como tinha respondido aos estímulos e se os tinha ou não nomeado. A entrevista poderia permitir verificar se e como os(as) participantes verbalizariam o procedimento utilizado para relacionar os estímulos durante o treino (Fase I, na qual havia reforçamento explícito para as escolhas, e Fase II, na qual foi introduzido o procedimento DOR com um formato de treino de pareamento consistente de estímulos modelos complexos e de comparação simples). A verbalização de como o(a) participante procedeu para responder aos estímulos indicaria a existência ou não de correspondência entre esse responder e o desempenho nas sessões, bem como o possível efeito da natureza dos estímulos experimentais.

Em seguida à entrevista, o experimentador entregava ao/ à participante um conjunto de figuras desenhadas em cartões plastificados, correspondentes aos estímulos apresentados no computador, e dizia-lhe: "Tente agora agrupar as figuras e cores usadas no computador de acordo com o modo pelo qual você executou a tarefa". A disponibilidade dos estímulos em cartões e a apresentação dessa instrução poderiam levar os participantes a reproduzir ou não a formação das mesmas classes organizadas nas sessões experimentais.

\section{Fase III: treino misto e testes}

Nesta fase, foi programado o treino misto das relações AB-E, AB-F, AD-C e AD-F, seguido da reapresentação dos testes E-F, F-E, C-F, F-C, B-D e D-B. Estas relações eram as mesmas da Fase 2, porém foram estabelecidas separadamente com desmembramento de AB-E/F em AB-E e AB-F. O de- 
sempenho dos participantes nos testes serviria para indicar se o controle sobre o mesmo foi restrito ou abrangente. $\mathrm{O}$ objetivo desta fase foi avaliar se, após a simplificação do treino com os modelos complexos, os participantes passariam a responder corretamente aos testes, demonstrando o controle de todos os componentes do modelo e, como consequiência, promovendo a emergência das novas relações de transitividade e de equivalência (Apêndice).

\section{Resultados e Discussão}

A Tabela 1 apresenta o desempenho dos quatro participantes no treino de discriminações condicionais e nos testes de relações emergentes. Como indica essa Tabela, todos os sujeitos responderam com $100 \%$ de acertos nos treinos AB$\mathrm{E} / \mathrm{F}$ e $\mathrm{AD}-\mathrm{C} / \mathrm{F}$, aos quais seguiu-se a aplicação dos testes das relações emergentes $\mathrm{EF}, \mathrm{FE}, \mathrm{CF}, \mathrm{FC}, \mathrm{BD}$ e DB, relacionadas a esses treinos. Dois participantes, CCR e FCN, responderam com $100 \%$ de acertos em todos os testes. Dois outros participantes, ASG e RMP, apresentaram uma porcentagem de acerto variável nos testes. O primeiro, ASG, apresentou 33\% de acerto nos testes EF e FE relacionados ao treino AB-E/F. Após o treino AD-C/F, ele apresentou $0 \%$ de acerto nos testes $\mathrm{CF}$ e FC. Nos testes BD e DB, relativos aos treinos AB-E/ $\mathrm{F}$ e AD-C/F, este participante obteve $33 \%$ de acerto. O outro participante, RMP, apresentou $66 \%$ e $16 \%$ de acerto, respectivamente, nos testes EF e FE; $100 \%$ de acerto nos testes $\mathrm{CF}$ e FC, e $0 \%$ de acerto nos testes BD e DB. Após o desmembramento dos treinos $\mathrm{AB}-\mathrm{E} / \mathrm{F}$ e $\mathrm{AD}-\mathrm{C} / \mathrm{F}$ em $\mathrm{AB}-\mathrm{E}$ e AB-F (ver Tabela 1), todos os participantes novamente demonstraram essas discriminações condicionais, respondendo com $100 \%$ de acerto. Nos testes EF, FE, CF, FC, BD e DB, os participantes ASG e RMP também apresentaram uma porcentagem de acerto variável, como ocorreu nestes testes antes do desmembramento dos treinos AB-E/F e AD-C/F. Nos teste EF e FE, respectivamente, ASG apresentou $100 \%$ e $83 \%$ de acerto; nos demais testes, CF, FC, BD e DB, o seu desempenho foi de $33 \%$ de acerto. O outro participante, RMP, apresentou $100 \%$ de acerto em todos os testes, exceto nos testes $\mathrm{BD}$ e $\mathrm{DB}$; nestes, sua porcentagem de acerto foi 0 . Os demais participantes, CCR e FCN, atingiram $100 \%$ de acerto em todos os testes.
$\mathrm{O}$ arranjo experimental implementado no estudo, ou seja, o treino das discriminações condicionais $\mathrm{AB}-\mathrm{E} / \mathrm{F}$ e $\mathrm{AD}-\mathrm{C} / \mathrm{F}$ com estímulos modelos complexos e estímulos de comparação simples, mostrou-se eficiente para o treino das discriminações condicionais, mas não foi igualmente eficiente para todos os participantes em promover as relações emergentes que foram testadas. Todos os participantes do estudo formaram as discriminações condicionais treinadas, mas apenas dois deles demonstraram todas as relações emergentes, indicando ter havido equivalência entre os estímulos usados no estudo. Após ter sido feito o desmembramento dos treinos $\mathrm{AB}-\mathrm{E} / \mathrm{F}$ e $\mathrm{AD}-\mathrm{C} / \mathrm{F}$ em $\mathrm{AB}-\mathrm{E}$ e $\mathrm{AB}-\mathrm{F}$, verificou-se que todos os participantes demonstraram as relações condicionais verificadas anteriormente. A novidade é que, nos testes $\mathrm{EF}$ e FE, pós-desmembramento dos treinos referidos, a porcentagem de acerto que tinha sido baixa nos mesmos testes prédesmembramento, aumentou consideravelmente (ver Tabela 1), de $33 \%$ para $100 \%$ e $83 \%$ (participante ASG), e de $66 \%$ e $16 \%$ para $100 \%$ nestes mesmos dois testes (participante RMP).

Os resultados indicam que o desmembramento dos treinos em blocos separados proporcionou um aumento no controle pelos dois componentes do modelo em dois participantes, sendo, portanto, um procedimento mais adequado para reverter o controle restrito de estímulo (cf. Dube \& McIlvane, 1999).

Nos testes das relações emergentes prédesmembramento dos treinos, os participantes ASG e RMP demonstraram uma maior dificuldade em responder corretamente a estímulos dos conjuntos B, C, D, E e F, tanto nas tentativas em que funcionavam como modelos quanto nas tentativas em que eram estímulos de comparação. Essa dificuldade foi superada parcialmente por ASG, após o desmembramento dos treinos em $\mathrm{AB}-\mathrm{E}$ a $\mathrm{AB}-\mathrm{F}$, quando essa participante foi exposta aos testes que envolviam alguns desses conjuntos de estímulos (E e F), mostrando um aumento na porcentagem de acertos. Porém, a porcentagem de acertos de ASG nos testes que envolviam os demais conjuntos de estímulos (C, B e D), após o desmembramento, permaneceu baixa. O participante RMP, não obstante ter superado, também, sua dificuldade em responder corretamente aos estímulos dos conjuntos B, C, D, E e F, ainda manteve dificuldade em responder aos conjuntos de estímulos $\mathrm{B}$ e $\mathrm{D}$, envolvidos

Tabela 1

Porcentagem de acertos dos participantes ASG, CCR, RMP e FCN no treino de discriminações condicionais e nos testes de relações emergentes

\begin{tabular}{|c|c|c|c|c|c|c|c|c|c|c|c|c|c|c|c|c|c|c|}
\hline Sujeitos & $\mathrm{AB}-\mathrm{E} / \mathrm{F}$ & EF & $\mathrm{FE}$ & $\mathrm{AD}-\mathrm{C} / \mathrm{F}$ & CF & $\mathrm{FC}$ & BD & DB & AB-E & AB-F & EF & $\mathrm{FE}$ & AD-C & AD-F & CF & $\mathrm{FC}$ & BD & DB \\
\hline ASG & 100 & 33 & 33 & 100 & 0 & 0 & 33 & 33 & 100 & 100 & 100 & 83 & 100 & 100 & 33 & 33 & 33 & 33 \\
\hline $\mathrm{CCR}$ & 100 & 100 & 100 & 100 & 100 & 100 & 100 & 100 & 100 & 100 & 100 & 100 & 100 & 100 & 100 & 100 & 100 & 100 \\
\hline RMP & 100 & 66 & 16 & 100 & 100 & 100 & 0 & 0 & 100 & 100 & 100 & 100 & 100 & 100 & 100 & 100 & 0 & 0 \\
\hline $\mathrm{FCN}$ & 100 & 100 & 100 & 100 & 100 & 100 & 100 & 100 & 100 & 100 & 100 & 100 & 100 & 100 & 100 & 100 & 100 & 100 \\
\hline
\end{tabular}

Nota

Os treinos AB-E e AB-F foram desdobramentos do treino AB-E/F

Os treinos AD-C e AD-F foram desdobramentos do treino AD-C/F 
nos testes pós-desmembramento dos estímulos de comparação (ver Tabela 1). É razoável supor que o desempenho de ASG e RMP nos testes não tenha decorrido do procedimento de desmembramento dos estímulos de comparação, mas em função de outra variável. Além da estrutura de treino de pareamento consistente, a utilização do mesmo tipo de estímulos visuais deste experimento, tanto como modelos quanto como estímulos de comparação, com atraso na apresentação daqueles, produziria um desempenho nos testes diferente do demonstrado pelos participantes ASG e RMP, isto é, a formação de relações emergentes? Estudos mostram que o atraso do modelo complexo é eficiente para produzir controle discriminativo das respostas (Stromer et al., 1993; Gutowski, Geren, Stromer \& Mackay, 1995).

Os resultados do presente estudo indicam que a natureza dos estímulos pode ter contribuído para o desempenho demonstrado por CCR e FCN nos treinos das discriminações condicionais aos quais foram submetidos (antes e após o desmembramento), bem como nos testes das relações emergentes. O papel da natureza dos estímulos no desempenho, a partir da análise do relato verbal em especial desses participantes, será considerado a seguir, ao serem apresentados e discutidos os dados das entrevistas pós-experimentais e os dados de manipulação dos estímulos impressos em cartões.

Enquanto respondia aos estímulos, a participante ASG relatou que tentava estabelecer associações entre eles; quando uma dada associação era correta, formava então uma regra que facilitava memorizar essas associações. Essa "estratégia" (a formação de regra) pode ter sido eficiente para o seu desempenho correto nos treinos (100\% de acerto), como ocorreu em outros estudos, dentre os quais o de Baptista e Assis (1995), Damim et al. (1998), Barros et al. (1998), que utilizaram um formato de treino de pareamento consistente de estímulos (modelos e de comparação usuais simples). Porém, essa "estratégia" não pareceu eficiente para o desempenho demonstrado na maioria dos testes (desempenho aquém de 50\% de acerto). A memorização, por exemplo, do elemento cor do modelo complexo decorreu da natureza dessa dimensão do estímulo, pois, segundo essa participante, "era mais fácil de lembrar". A memorização de estímulos experimentais é passível de ocorrer quando se tenta estabelecer a relação entre alguma dimensão do estímulo e algum aspecto do cotidiano, enquanto um recurso que facilite a formação de classes. É provável que, devido à sua natureza, estímulos experimentais como os utilizados no presente estudo propiciem esse tipo de relação (ver relatos pós-experimentais de alguns participantes do estudo de Baptista \& Assis, 1995, e discussão de Barros, Baptista, \& Assis, 1998).

Os participantes CCR, RMP e FCN verbalizaram que a conseqüência reforçadora ("bip") disponível para suas respostas de escolha propiciou-lhes acelerar o seu desempenho após esse controle ter-se verificado (ver Fase I), o que mostra o efeito de um dos tipos de conseqüência reforçadora para acertos. Segundo o participante RMP, o "bip", além disso, permitiu-lhe estabelecer associações entre os estímulos ("associações de imagens, criando historinhas", o que pode ser considerado como formação de regras). Quanto à resposta ao modelo complexo (ver Fase II e III), esse participante expressou que respondia ao elemento que era idêntico ao estímulo de comparação apresentado na tela (em conjunto com os demais). Os participantes CCR e FCN, entretanto, disseram que respondiam, respectivamente, de forma aleatória e arbitrária a um dos elementos do modelo - um dado verbal leva a supor que esses dois participantes se equivocaram em relatar o desempenho que de fato demonstraram nas sessões durante os treinos de relações condicionais (nos quais obtiveram $100 \%$ de acertos).

A participante ASG expressou ter nomeado alguns estímulos isoladamente e outros em sua relação com os estímulos com os quais eram pareados. A participante CCR verbalizou que, ao nomear os estímulos, procurava relacionálos com a cor do modelo. RMP expressou que nomeou apenas alguns estímulos, de acordo com as associações (de imagens, já mencionadas), que ele estabeleceu entre estímulos. A participante FCN também verbalizou o uso da nomeação de estímulos criando associações entre eles, de fácil memorização. $\mathrm{O}$ recurso à nomeação, por parte de todos os participantes, deve ser considerado um indicativo do efeito da natureza dos estímulos (em particular, da dimensão cor, no caso da participante CCR, ou de alguma outra dimensão, no caso dos demais participantes).

Os participantes ASG e RMP manipularam os cartões relacionando os estímulos de modo diferente de como o fizeram nas sessões experimentais, enquanto que os dois outros participantes, CCR e FCN, incluíram os estímulos em classes de forma coerente com o desempenho nas sessões. A manipulação dos estímulos impressos nos cartões possibilitou que todos os participantes voltassem a ter contato com estímulos correspondentes àqueles apresentados no computador, constituindo-se numa espécie de pós-testes. A oportunidade de manipulação dos estímulos foi uma forma de avaliar se os participantes com um desempenho bem-sucedido nos testes manteriam o mesmo desempenho fora da situação experimental. Pode-se dizer que isso de fato ocorreu, pois, ao contrário dos outros dois, os participantes (CCR e FCN) que apresentaram $100 \%$ de acerto em todos os testes foram precisamente os que conseguiram reproduzir, após as sessões, a formação de classes de estímulos.

Este estudo aponta algumas questões relevantes, além da apontada acima. Uma questão refere-se ao desempenho dos participantes na Fase II. Podem os resultados ser atribuídos ao procedimento de treino de pareamento consistente? Esses resultados são decorrentes da aplicação do reforçamento diferencial na Fase I? Devem-se à interação de ambos os procedimentos? Se, na Fase I, os estímulos exerceram um controle independente sobre o responder, pode-se evidenciar a eficácia deste procedimento de treino para a emergência de relações equivalentes entre estímulos. Contudo, é admissível que, na Fase II, os participantes estivessem respondendo à situação de teste, com insensibilidade à consistência na apresentação dos estímulos, mas seu desempenho poderia estar sendo afetado por sua história prévia com esses estímulos. Se esta suposição está correta, deve-se então questionar se treinando diretamente A-F e B-E, seria possí- 
vel demonstrar a relação AB-E/F, por exemplo. Para responder a esta questão seria necessário modificar o arranjo de treino da Fase I, de modo a garantir que a única variável de controle fosse o procedimento de treino de pareamento consistente, substituindo a contingência por este tipo de arranjo de treino desde o início do estudo.

Outra questão refere-se ao número de exposições aos blocos de treino. Talvez fosse necessário um maior número de tentativas por bloco, como foi observado com a participante ASG. Contudo, o que merece maior destaque neste ponto é a reexposição ao bloco de treino, indicando que a escolha apresentada pelo(a) participante não estava correta se a exposição fosse prolongada. A repetição poderia funcionar como uma pista para que o participante discriminasse que ele não estava avançando no treino e, portanto, continuava errando, sendo necessário mudar sua escolha. Uma outra forma de avaliar este procedimento, na ausência de conseqüências diferenciais explícitas, seria limitar o número de tentativas para uma única exposição, independentemente do resultado obtido, de modo a avaliar se o procedimento de treino de pareamento consistente seria eficaz, isoladamente, para controlar esse tipo de resposta. No entanto, um participante submetido a este procedimento pode não ter a oportunidade de perceber o controle pelo tipo de emparelhamento entre o modelo e o estímulo de comparação consistente.

As questões mencionadas poderão ser respondidas em estudos futuros que continuarão investigando os efeitos do treino de pareamento consistente de estímulos no estabelecimento de discriminações condicionais e na emergência de novas relações. No presente estudo, os resultados sugerem que é possível a formação de relações condicionais entre estímulos complexos e que estes estímulos podem tornar-se equivalentes entre si, a partir de um formato de treino de pareamento consistente, sem conseqüências diferenciais imediatas em cada tentativa. A sugestão dessa possibilidade é importante porque abre, como perspectiva, a discussão a respeito do papel do reforço como tradicionalmente tem sido usado nos estudos sobre a formação de discriminações condicionais envolvendo estímulos complexos. Levanta, ainda, uma questão teórica, metodológica e socialmente relevante, que se refere ao forte controle passível de ser estabelecido pelos estímulos discriminativos e condicionais sob condições apropriadas e na ausência de conseqüências diferenciais imediatas. No presente estudo, os resultados indicam que o formato de treino sem consequiências diferenciais imediatas é extensivo a estímulos complexos e não apenas a estímulos simples como já foi documentado pela literatura (ver, dentre outros, os estudos de Baptista \& Assis, 1995; Assis et al., 1997; Assis et al., 2000; Álvares et al., 2001).

\section{Agradecimentos}

Os autores desejam agradecer ao prof. Dr. Joseph Spradlin e Dra. Ruth Rehfeldt pelas sugestões, ao prof. Dr. W. Lee Martin pelos comentários e a Ane Margarete Monte Verde Silva pelo desenvolvimento do programa de controle de apresentação das contingências experimentais e registro de dados comportamentais.

\section{Referências}

Álvares, S. M. M., Assis, G. J. A., Sampaio, M. E. C., \& Esteves, I. P. (2001). Discriminações condicionais sem conseqüências diferenciais em crianças: efeitos da história de treino precoce de simetria. Estudos de Psicologia (Natal), 6(1), 7-20.

Assis, G. J. A., Baptista, M. Q. G., Damin, E. T. B., \& Álvares, S. M. M. (1997). Consistency training and equivalence relations without differential consequences. Experimental Analysis of Human Behavior Bulletin, 15(1), 6-7.

Assis, G. J. A., Baptista, M. Q. G., Kato, O. M. K., \& Alves, K. R. (2000). Relações de equivalência após treino consistente de estímulos sob controle contextual. Psicologia: Teoria e Pesquisa, 16(2) 125-133.

Assis, G. J. A., Baptista, M. Q. G., Kato, O. M., \& Cardoso, D. G. (2003). Equivalência de estímulos após treino de pareamento consistente de estímulos com atraso do modelo. Estudos de Psicologia (Natal), 8(1), 63-73.

Assis, G. J. A., Kato, O. M., Menezes, A. B., \& Lopes Junior, J. (2000, outubro). Complex stimuli equivalence after consistent matching among sample-correct comparison: effect of the differential observing response. Trabalho apresentado no $5^{\mathrm{o}}$ International Congress on Behaviorism and Sciences of Behavior, Xalapa, Mexico.

Baptista, M. Q. G., \& Assis, G. J. A. (1995). Treino por consistência de estímulos sem consequiências diferenciais. Psicologia: Teoria e Pesquisa, 11(3), 173-179.

Barros, C. W. L., Baptista M. Q. G., \& Assis, G. J. A. (1998). Efeitos de história de treino sobre a formação de classes de estímulos equivalentes. Acta Comportamentalia, 6(2), 111-128.

Constantine, B., \& Sidman, M. (1975). Role of naming in delayed matching to sample. American Journal of Mental Deficiency, 6, 680-689.

Damim, E. T. B., Assis, G. J. A., \& Baptista, M. Q. G. (1998). Efeitos da distribuição treino/testes sobre a formação de classes de estímulos equivalentes sem conseqüências diferenciais. Psicologia: Teoria e Pesquisa, 14(1), 41-49.

Dube, W. V. (1996). Teaching discriminations skills to persons with mental retardation. Temas em Educação Especial (São Carlos, SP), 3, 73-96.

Dube, W. V., \& MacIvane, W. J. (1999). Reduction of stimulus overselectivity with nonverbal diferential observing responses. Journal of Applied Behavior Analysis, 32, 25-33.

Gutowski, S. J., Geren, M., Stromer, R., \& Mackay, H. (1995). Restricted stimulus control in delayed matching to complex samples: a preliminary analysis of the role of naming. Experimental Analysis of Human Behavior Bulletin, 13, 18-24.

Lopes Junior, J., Costa, G. G., Gonsales, L. F. S., \& Golfeto, R. M. (2001). Aprendizagem e emergência de relações condicionais com estímulos modelos complexos. In H. J. Guilhardi (Org.), Sobre comportamento e cognição: expondo a variabilidade (pp. 401-421). Santo André, SP: ESETec Editores Associados.

Rehfeldt, R. A., Clayton, M., \& Hayes, L. J. (1998). Blocking the formation of 5 -member equivalence classes using complex samples. Mexican Journal of Behavior Analysis, 24(3), 279-292.

Sidman, M. (1992). Equivalence relations: some basic considerations. In S. C. Hayes (Org.), Understanding verbal relations: the second and third international institute on verbal relations (pp. 15-27). Reno, Nevada: Context.

Stikeleather, G., \& Sidman, M. (1990). An instance of spurious equivalence relations. The Analysis of Verbal Behavior, 8, 1-11.

Stromer, R., McIlvane, W. J., Dube, W. V., \& Mackay, H. A. (1993). Assessing control by elements of complex stimuli in delayed matching to sample. Journal of the Experimental Analysis of Behavior, 59(1), 83-102.

Stromer, R., McIlvane, W. J., \& Serna, R. W. (1993). Complex stimulus control and equivalence. The Psychological Record, 43, 585-598.

Stromer, R., \& Stromer, J. B. (1990). The formation of arbitrary stimulus classes in matching to complex samples. The Psychological Record, 40, 51-66. 
1 Para a identificação do controle pelos elementos do estímulo complexo, tem sido citada na literatura a notação CSS. A letra "C" indica um modelo "complexo" (com mais de um elemento) e a letra "S" estímulo de comparação "simples".

Grauben Assis, doutor em Educação pela Universidade Federal de São Carlos, SP, é bolsista de Produtividade do CNPq e professor no departamento de Psicologia Experimental e no Programa de Pós-Graduação em Teoria e Pesquisa do Comportamento da Universidade Federal do Pará. Endereço para correspondência: Travessa Castelo Branco, 1923/301 (bairro do Guamá); Belém, PA; CEP 66063-420; Telefax: 55 (91) 2111662.E-mail: gjaa@cpgp.ufpa.br

Marcelo Galvão Baptista, doutor em Educação pela Universidade Federal de São Carlos, SP, é professor no departamento de Psicologia Experimental e no Programa de Pós-Graduação em Teoria e Pesquisa do Comportamento da Universidade Federal do Pará.

Olivia Misae Kato, doutora em Psicologia Experimental pela Universidade de São Paulo, é professora no departamento de Psicologia Experimental e no Programa de Pós-Graduação em Teoria e Pesquisa do Comportamento da Universidade Federal do Pará.

Aline Beckmann de Menezes foi bolsista de iniciação científica do PIBIC-CNPq e é mestranda no Programa de Pós-Graduação em Teoria e Pesquisa do Comportamento da Universidade Federal do Pará. 


\section{Apêndice}

Treino de relações condicionais com reforçamento diferencial (RF - Fase I) e com pareamento consistente (PC - Fases II e III); testes de relações emergentes com o número e tipo de tentativas.

\begin{tabular}{|c|c|c|c|c|c|c|}
\hline \multirow[t]{2}{*}{ Fase * } & \multirow[t]{2}{*}{ Relação } & \multirow[t]{2}{*}{ Tipo de tentativas } & \multicolumn{2}{|c|}{ Número de tentativas } & \multirow[t]{2}{*}{ RD } & \multirow[t]{2}{*}{ PC } \\
\hline & & & Parcial & Total & & \\
\hline \multirow{18}{*}{$\begin{array}{l}\text { Fase I } \\
\text { (treino) }\end{array}$} & \multirow{3}{*}{$\begin{array}{l}\text { Treino AF } \\
\text { (aquisição) }\end{array}$} & A1 F1(+)F2F3 & 12 & & $\mathrm{X}$ & - \\
\hline & & $\mathrm{A} 2 \mathrm{~F} 2(+) \mathrm{F} 1 \mathrm{~F} 3$ & 12 & & $\mathrm{X}$ & - \\
\hline & & A3 F3(+)F1F2 & 12 & 36 & $\mathrm{X}$ & - \\
\hline & \multirow{3}{*}{$\begin{array}{c}\text { Treino misto } \\
\mathrm{AF}^{*}\end{array}$} & $\mathrm{~A} 1 \mathrm{~F} 1(+) \mathrm{F} 2 \mathrm{~F} 3$ & 02 & & $\mathrm{X}$ & - \\
\hline & & A2 F2(+)F1F3 & 02 & & $\mathrm{X}$ & - \\
\hline & & A3 F3(+)F1F2 & 02 & 06 & $\mathrm{X}$ & - \\
\hline & \multirow{3}{*}{$\begin{array}{l}\text { Treino BE } \\
\text { (aquisição) }\end{array}$} & B1 E1(+)E2E3 & 12 & & $\mathrm{X}$ & - \\
\hline & & $\mathrm{B} 2 \mathrm{E} 2(+) \mathrm{E} 1 \mathrm{E} 3$ & 12 & & $\mathrm{X}$ & - \\
\hline & & B3 E3(+)E1E2 & 12 & 36 & $\mathrm{X}$ & - \\
\hline & \multirow{3}{*}{$\begin{array}{l}\text { Treino misto } \\
\text { BE* }\end{array}$} & B1 E1(+)E2E3 & 02 & & $\mathrm{X}$ & - \\
\hline & & B2 E2(+)E1E3 & 02 & & $\mathrm{X}$ & - \\
\hline & & B3 E3(+)E1E2 & 02 & 06 & $\mathrm{X}$ & - \\
\hline & \multirow{3}{*}{ Treino DC } & $\mathrm{D} 1 \mathrm{C} 1(+) \mathrm{C} 2 \mathrm{C} 3$ & 12 & & $\mathrm{X}$ & - \\
\hline & & $\mathrm{D} 2 \mathrm{C} 2(+) \mathrm{C} 1 \mathrm{C} 3$ & 12 & & $\mathrm{X}$ & - \\
\hline & & D3 $\mathrm{C} 3(+) \mathrm{C} 1 \mathrm{C} 2$ & 12 & 36 & $\mathrm{X}$ & - \\
\hline & \multirow{3}{*}{$\begin{array}{c}\text { Treino misto } \\
\text { DC* }\end{array}$} & $\mathrm{D} 1 \mathrm{C} 1(+) \mathrm{C} 2 \mathrm{C} 3$ & 02 & & $\mathrm{X}$ & - \\
\hline & & $\mathrm{D} 2 \mathrm{C} 2(+) \mathrm{C} 1 \mathrm{C} 3$ & 02 & & $\mathrm{X}$ & - \\
\hline & & D3 $\mathrm{C} 3(+) \mathrm{C} 1 \mathrm{C} 2$ & 02 & 06 & $\mathrm{X}$ & - \\
\hline \multirow{18}{*}{$\begin{array}{l}\text { Fase II } \\
\text { (treino e } \\
\text { testes) }\end{array}$} & \multirow{6}{*}{$\begin{array}{c}\text { Treino } \\
\text { AB-E/F } \\
\text { (aquisição) }\end{array}$} & A1B1 E1(+)E2E3(E4)** & 12 & \multirow{2}{*}{24} & - & $\mathrm{X}$ \\
\hline & & A1B1 F1(+)F2F3(F4)** & 12 & & - & $\mathrm{X}$ \\
\hline & & A2B2 E2(+)E1E3(E4)** & 12 & \multirow{2}{*}{24} & - & $\mathrm{X}$ \\
\hline & & $\mathrm{A} 2 \mathrm{~B} 2 \mathrm{~F} 2(+) \mathrm{E} 1 \mathrm{~F} 3(\mathrm{~F} 4)^{* *}$ & 12 & & - & $\mathrm{X}$ \\
\hline & & A3B3 E3(+)E1E2(E4)** & 12 & \multirow[b]{2}{*}{24} & - & $\mathrm{X}$ \\
\hline & & A2B3 F3(+)F1F2(F4)** & 12 & & - & $\mathrm{X}$ \\
\hline & \multirow{6}{*}{$\begin{array}{l}\text { Treino misto } \\
\text { AB-E/F } \\
\text { (linha de base) }\end{array}$} & A1B1 E1(+)E2E3(E4)** & 02 & \multirow[b]{6}{*}{12} & - & $\mathrm{X}$ \\
\hline & & A1B1 F1(+)F2F3(F4)** & 02 & & - & $\mathrm{X}$ \\
\hline & & $\mathrm{A} 2 \mathrm{~B} 2 \mathrm{E} 2(+) \mathrm{E} 1 \mathrm{E} 3(\mathrm{E} 4) * *$ & 02 & & - & $\mathrm{X}$ \\
\hline & & A2B2 F2(+)F1F3(F4)** & 02 & & - & $\mathrm{X}$ \\
\hline & & A3B3 E3(+)E1E2(E4)** & 02 & & - & $\mathrm{X}$ \\
\hline & & A3B3 F3(+)F1F2(F3)** & 02 & & - & $\mathrm{X}$ \\
\hline & \multirow{3}{*}{ Teste E-F } & E1 F1(+)F2F3 & 02 & \multirow[b]{3}{*}{06} & - & - \\
\hline & & E2 F2(+)F3F1 & 02 & & - & - \\
\hline & & E3 F3(+)F2 F1 & 02 & & - & - \\
\hline & \multirow{3}{*}{ Teste F-E } & F1 E1(+)E2E3 & 02 & \multirow{3}{*}{06} & - & - \\
\hline & & F2 E2(+)E3E1 & 02 & & - & - \\
\hline & & F3 E3(+)E2E1 & 02 & & - & - \\
\hline
\end{tabular}


Apêndice (continuação)

Treino de relações condicionais com reforçamento diferencial (RF - Fase I) e com pareamento consistente (PC - Fases II e III); testes de relações emergentes com o número e tipo de tentativas.

\begin{tabular}{|c|c|c|c|c|c|c|}
\hline \multirow[t]{2}{*}{ Fase * } & \multirow[t]{2}{*}{ Relação } & \multirow[t]{2}{*}{ Tipo de tentativas } & \multicolumn{2}{|c|}{ Número de tentativas } & \multirow[t]{2}{*}{ RD } & \multirow[t]{2}{*}{ PC } \\
\hline & & & Parcial & Total & & \\
\hline \multirow{24}{*}{$\begin{array}{l}\text { Fase II } \\
\text { (treino e } \\
\text { testes) }\end{array}$} & \multirow{6}{*}{$\begin{array}{l}\text { Treino } \\
\text { AD-C/F } \\
\text { (aquisição) }\end{array}$} & $\mathrm{A} 1 \mathrm{D} 1 \mathrm{C} 1(+) \mathrm{C} 2 \mathrm{C} 3(\mathrm{C} 4)^{* *}$ & 12 & \multirow[b]{2}{*}{24} & - & $\mathrm{X}$ \\
\hline & & A1D1 F1(+)F2F3(F4)** & 12 & & - & $\mathrm{X}$ \\
\hline & & $\mathrm{A} 2 \mathrm{D} 2 \mathrm{C} 2(+) \mathrm{C} 1 \mathrm{C} 3(\mathrm{C} 4)^{* *}$ & 12 & \multirow{2}{*}{24} & - & $\mathrm{X}$ \\
\hline & & A2D2 F2(+)F1F3(F4)** & 12 & & - & $\mathrm{X}$ \\
\hline & & $\mathrm{A} 3 \mathrm{D} 3 \mathrm{C} 3+\mathrm{C} 1 \mathrm{C} 2(\mathrm{C} 4)^{* *}$ & 12 & \multirow{2}{*}{24} & - & $\mathrm{X}$ \\
\hline & & A3D3 F3 + F1F2 (F3)** & 12 & & - & $\mathrm{X}$ \\
\hline & \multirow{6}{*}{$\begin{array}{c}\text { Treino misto } \\
\mathrm{AD}-\mathrm{C} / \mathrm{F} \\
\text { (linha de } \\
\text { base) }\end{array}$} & A1D1 C1(+)C2C3(C4)** & 02 & \multirow{6}{*}{12} & - & $\mathrm{X}$ \\
\hline & & A1D1 F1(+)F2F3(F4)** & 02 & & - & $\mathrm{X}$ \\
\hline & & $\mathrm{A} 2 \mathrm{D} 2 \mathrm{C} 2(+) \mathrm{C} 1 \mathrm{C} 3(\mathrm{C} 4)^{* *}$ & 02 & & - & $\mathrm{X}$ \\
\hline & & A2D2 F2(+)F1F3(F4)** & 02 & & - & $\mathrm{X}$ \\
\hline & & A3D3 C3 +C1C2(C4)** & 02 & & - & $\mathrm{X}$ \\
\hline & & A3D3 F3 + F1F2 (F4)** & & & - & $\mathrm{X}$ \\
\hline & \multirow{3}{*}{ Teste CF } & $\mathrm{C} 1 \mathrm{~F} 1(+) \mathrm{F} 2 \mathrm{~F} 3$ & 02 & & - & - \\
\hline & & $\mathrm{C} 2 \mathrm{~F} 2(+) \mathrm{F} 1 \mathrm{~F} 3$ & 02 & & - & - \\
\hline & & $\mathrm{C} 3 \mathrm{~F} 3(+) \mathrm{F} 2 \mathrm{~F} 1$ & 02 & 06 & - & - \\
\hline & \multirow{3}{*}{ Teste FC } & $\mathrm{F} 1 \mathrm{C} 1(+) \mathrm{C} 2 \mathrm{C} 3$ & 02 & & - & - \\
\hline & & $\mathrm{F} 2 \mathrm{C} 2(+) \mathrm{C} 1 \mathrm{C} 3$ & 02 & & - & - \\
\hline & & $\mathrm{F} 3 \mathrm{C} 3(+) \mathrm{C} 2 \mathrm{C} 1$ & 02 & 06 & - & - \\
\hline & \multirow{3}{*}{ Teste BD } & B1 D1(+)D2 D3 & 02 & & - & - \\
\hline & & B2 D2(+)D1D3 & 02 & & - & - \\
\hline & & B3 D3(+)D2 D1 & 02 & 06 & - & - \\
\hline & \multirow{3}{*}{ Teste DB } & $\mathrm{D} 1 \mathrm{~B} 1(+) \mathrm{B} 2 \mathrm{~B} 3$ & 02 & & - & - \\
\hline & & $\mathrm{D} 2 \mathrm{~B} 2(+) \mathrm{B} 1 \mathrm{~B} 3$ & 02 & & - & - \\
\hline & & D3 B3(+)B2 B1 & 02 & 06 & - & - \\
\hline \multirow{24}{*}{$\begin{array}{l}\text { Fase III } \\
\text { (treino e } \\
\text { testes) }\end{array}$} & \multirow{6}{*}{$\begin{array}{l}\text { Treino misto } \\
\text { AB-E/F (AB- } \\
\text { E e AB-F) } \\
\text { (linha de } \\
\text { base) }\end{array}$} & A1B1 E1(+)E2E3(E4)** & 04 & \multirow{5}{*}{12} & - & $\mathrm{X}$ \\
\hline & & A2B2 E2(+)E3E1(E4)** & 04 & & - & $\mathrm{X}$ \\
\hline & & A3B3 E3(+)E1E2(E4)** & 04 & & - & $\mathrm{X}$ \\
\hline & & A1B1 F1(+)F2F3(F4)** & 04 & & - & $\mathrm{X}$ \\
\hline & & $\mathrm{A} 2 \mathrm{~B} 2 \mathrm{~F} 2(+) \mathrm{F} 3 \mathrm{~F} 1(\mathrm{~F} 4)^{* *}$ & 04 & & - & $\mathrm{X}$ \\
\hline & & A3B3 F3(+)F1F2(F4)** & 04 & 12 & - & $\mathrm{X}$ \\
\hline & \multirow{6}{*}{$\begin{array}{c}\text { Treino misto } \\
\text { AD-C/F (AD- } \\
\text { C e AD-F) } \\
\text { (linha de } \\
\text { base) }\end{array}$} & $\mathrm{A} 1 \mathrm{D} 1 \mathrm{C} 1(+) \mathrm{C} 2 \mathrm{C} 3(\mathrm{C} 4)^{* *}$ & 04 & & - & $\mathrm{X}$ \\
\hline & & $\mathrm{A} 2 \mathrm{D} 2 \mathrm{C} 2(+) \mathrm{C} 3 \mathrm{C} 1(\mathrm{C} 4)^{* *}$ & 04 & & - & $\mathrm{X}$ \\
\hline & & $\mathrm{A} 3 \mathrm{D} 3 \mathrm{C} 3(+) \mathrm{C} 1 \mathrm{C} 2(\mathrm{C} 4)^{* *}$ & 04 & 12 & - & $\mathrm{X}$ \\
\hline & & A1D1 F1 $(+) F 2 F 3(\mathrm{~F} 4) * *$ & 04 & & - & $\mathrm{X}$ \\
\hline & & $\mathrm{A} 2 \mathrm{D} 2 \mathrm{~F} 2(+) \mathrm{F} 3 \mathrm{~F} 1(\mathrm{~F} 4)^{* *}$ & 04 & & - & $\mathrm{X}$ \\
\hline & & A3D3 F3(+)F1F2(F4)** & 04 & 12 & - & $\mathrm{X}$ \\
\hline & & $\mathrm{E} 1 \mathrm{~F} 1(+) \mathrm{F} 2 \mathrm{~F} 3$ & 02 & & - & - \\
\hline & Teste EF & $\mathrm{E} 2 \mathrm{~F} 2(+) \mathrm{F} 3 \mathrm{~F} 1$ & 02 & & - & - \\
\hline & & E3 F3(+)F1F2 & 02 & 06 & - & - \\
\hline & & F1 E1(+)E2E3 & 02 & & - & - \\
\hline & Teste FE & F2 E2(+)E3E1 & 02 & & - & - \\
\hline & & F3 E3(+)E1E2 & 02 & 06 & - & - \\
\hline & & $\mathrm{C} 1 \mathrm{~F} 1(+) \mathrm{F} 2 \mathrm{~F} 3$ & 02 & & - & - \\
\hline & Teste CF & $\mathrm{C} 2 \mathrm{~F} 2(+) \mathrm{F} 3 \mathrm{~F} 1$ & 02 & & - & - \\
\hline & & $\mathrm{C} 3 \mathrm{~F} 3(+) \mathrm{F} 1 \mathrm{~F} 2$ & 02 & 06 & - & - \\
\hline & & $\mathrm{F} 1 \mathrm{Cl}(+) \mathrm{C} 2 \mathrm{C} 3$ & 02 & & - & - \\
\hline & Teste FC & $\mathrm{F} 2 \mathrm{C} 2(+) \mathrm{C} 3 \mathrm{C} 1$ & 02 & & - & - \\
\hline & & $\mathrm{F} 3 \mathrm{C} 3(+) \mathrm{C} 1 \mathrm{C} 2$ & 02 & 06 & - & - \\
\hline
\end{tabular}

(+) Estímulo consistente que corresponde ao tradicionalmente chamado correto.

* Nenhum dos treinos mistos (Fase I) foi utilizado como linha de base para os testes.

** Estímulos de distração

Nota. No treino AB-E/F e no treino AD-C/F (Fase II), foram programadas tentativas nas quais o primeiro elemento do modelo complexo era pareado com o respectivo estímulo de comparação consistente, sendo depois o segundo elemento do modelo pareado com o estímulo consistente. Por exemplo, o elemento A1 com $\mathrm{F} 1+$, nas tentativas do tipo A1B1 F1+ F2F3 (F4), e depois o elemento B1 com E1+, nas tentativas do tipo A1B1 E1+E2E3 (E4). Na tabela acima, as tentativas do tipo A1B1 F1(+) F2F3 (F4) constam após as do tipo A1B1 E1(+) E2E3 (E4), ao contrário da ordem seguida no treino. 